\title{
Eficacia de la hipnosis en la modificación de variables psicológicas y fisiológicas en deportistas
}

\section{Efficiency of Hypnosis in Psychological and Physiological Variables in Sportsman}

Recibido: enero 17 de 2012 | Revisado: junio 10 de 2012 | Aceptado: octubre 2 de 2012

\author{
RubÉN FERnÁNDEZ GARCÍA* \\ LAURA C. SÁNCHEZ SÁNCHEZ ** \\ Universidad de Almería, España \\ FÉLIX ZURITA ORTEGA **** \\ Universidad de Granada, España
}

doi:10.11144/Javeriana.UPSY12-2.ehmv

Para citar este artículo: Fernández, R., Sánchez, L. C. \& Zurita, F. (2013). Eficacia de la hipnosis en la modificación de variables psicológicas y fisiológicas en deportistas. Universitas Psychologica, 12(2), 483 491.

* Correspondencia concerniente a este a este artículo: Rubén Fernández García. Departamento de Enfermería y Fisioterapia, Universidad de Almería. La Cañada de San Urbano s/n, Almería (España). Teléfono: (34) 95-0014126.E-mail: rubenfer@ual.es

* Universidad de Almería, Facultad de Psicología, La Cañada de San Urbano s/n, Almería (España). Teléfono: (34) 95-0014128. E-mail: 1ss250@ual.es

${ }^{* * * *}$ Universidad de Granada, España, Facultad de Ciencias de la Educación. Campus de Cartuja. E- mail: felixzo@ugr.es

\section{RESUMEN}

En este estudio se evaluó la eficacia de la hipnosis en la modificación de un conjunto de variables psicológicas y fisiológicas durante la realización de pruebas de esfuerzo. Se utilizaron un total de 24 participantes ciclistas. El procedimiento consistió en enviar al azar a los participantes a un grupo experimental con hipnosis o al grupo control. En ambos grupos se midieron variables psicológicas y fisiológicas relacionadas con el deporte. Los resultados no mostraron diferencias estadísticamente significativas entregrupos en las variables evaluadas. Sí se observó una tendencia en el grupo experimental a puntuar más positivamente en varias variables psicológicas analizadas. Se concluye que sería de interés desarrollar futuras investigaciones ampliando el tamaño de la muestra y aplicar el procedimiento en diferentes modalidades deportivas.

Palabras clave autores

Hipnosis, psicosomática, deporte, variables físicas.

Palabras clave descriptores

Psicología del deporte, investigación cuantitativa, diseño cuasi-experimental

\section{A B S T R A C T}

In this paper evaluated the efficiency of hypnosis on modification of a set of psychological and physiological variables during the performance of a task force. There were in use a total of 24 cycle participants. The procedure consisted of sending at random to the participants to an experimental group with hypnosis and group control. In both groups there measured up psychological and physiological variables related to the sport. Results indicate that there was not a statistically significant difference between the two groups. However, a tendency more positively in a set of psychological variables was observed in the experimental group. It is concluded that it would be of interest to develop future researches extending the size of the sample and to apply the procedure in different sports modalities.

Key words authors

Hypnosis, Psychosomatic, Sport, Physical Variables.

Key words plus

Sport Psychology, Quantitative Research, Quasi-experimental Design. 


\section{Introducción}

Dentro del ámbito de deporte, sobre todo el relacionado con categorías infantiles, son varios los objetivos que se plantean, entre otros la búsqueda del bienestar físico y emocional por parte del practicante (Moreno, Conte, Borges \& González-Cutre, 2008). En categorías más avanzadas en edad, como el deporte juvenil y sobre todo el aficionado y profesional o de élite, las exigencias son mucho mayores y las expectativas cambian, pues comienza a cobrar mayor importancia la consecución de un buen resultado deportivo (Grindstaff \& Fisher, 2006). Con esta finalidad, para ayudar a deportistas de categorías "superiores" a obtener un buen rendimiento, se llevan utilizando desde hace años técnicas psicológicas que intentan ajustarse a las demandas contextuales (Lorenzo, Pujals, Navarro \& Lorenzo, 2012). En este sentido, también resulta de interés dar cuenta y descubrir las variables psicológicas predictoras de un buen rendimiento deportivo (García-Naveira \& Remor, 2011).

Siguiendo con lo expuesto, efectivamente son muchas y variadas las técnicas utilizadas dentro del contexto deportivo que intentan favorecer una óptima condición física y fisiológica, mediante la modificación de numerosas variables subjetivas con componente eminentemente psicológico como el dolor, la fatiga, la relajación, capacidad de concentración, etc. (Fernández, Secades, Terrados, García \& García, 2004; Lorenzo et al., 2012). Una de estas técnicas es la hipnosis, la cual desde un punto de vista científico no ha sido ampliamente utilizada en el ámbito deportivo. Por este motivo, es decir, la escasez de publicaciones sobre psicología y deporte en relación con otras temáticas tratadas en este campo, hemos planteado un estudio que verse sobre esta técnica y sus posibles beneficios, utilizando en este caso un procedimiento novedoso ajustado a las condiciones de los participantes.

De todas formas como se comentaba más arriba, a pesar de no existir un gran número de reseñas científicas, la hipnosis sí ha tenido su aplicación en deportes como el piragüismo (Fernández et al., 2004), criket (Barker \& Jones, 2006), voleibol (Barker, Jones \& Greenlees, 2010), baloncesto (Pates, Maynard \& Cummings, 2002), ciclismo (Lindsay, Maynard \& Thomas, 2005), etc., con la intención de intentar mejorar las capacidades deportivas mediante el manejo y modificación de distintas variables con componente psicológico como la tensión, el agarrotamiento, la fatiga, etc.

Por otro lado, dentro del numeroso conjunto de teorías que tratan de explicar el funcionamiento de la hipnosis (Kirenskaya, Novotosky \& Zvonikov, 2011; Landolt \& Milling, 2011; Raz, Uriel \& Shany, 2011), para desarrollar el procedimiento "hipnótico" en esta investigación se tomó como referencia el "enfoque alternativo", donde los "fenómenos" hipnóticos se explican como un proceso motivacionalmente inducido que tienen lugar por la adecuada manipulación de características psicológicas y psicosociales (Jara \& Martínez, 1999; Pérez, 1999). En este sentido, el procedimiento desarrollado basado en este enfoque, podría favorecer a priori en los participantes mejores puntuaciones en las variables de tipo psicológico evaluadas.

\section{Objetivo del estudio}

Siguiendo con lo expuesto, se plantea como objetivo principal evaluar la eficacia de la hipnosis en la modificación de una serie de variables psicológicas y fisiológicas registradas durante la realización de pruebas de esfuerzo, utilizando para la ocasión el Test de Entrenamiento por Vatios.

Atendiendo entonces al objetivo indicado arriba, se clarifica que la hipótesis de partida fue comprobar que la hipnosis sí es una técnica que favorece diferencias estadísticamente significativas en las variables fisiológicas: fatiga percibida, frecuencia cardiaca hasta no cumplir el criterio (mantenimiento de 70-80 pedaladas por minuto mientras se realiza la prueba de esfuerzo) y en las variables psicológicas: muy relajado-muy nervioso, controlado-sin control, muy enérgico-muy fatigado, concentrado-descentrado, sin esforzarse-con gran esfuerzo y, por último, grado de sugestionabilidad. 


\section{Método}

\section{Muestra}

La muestra estuvo compuesta de 24 ciclistas, 12 juveniles y 12 aficionados, con una edad media de 17.75 años (rango 16-21 años) y un promedio de 5.75 años compitiendo. Por motivos de accesibilidad, los participantes pertenecían al Club Ciclista Colloto (Asturias). El estudio cumplió con las normas éticas del Comité de Investigación y Declaración de Helsinki de 1975. Se contó en todos los casos con el consentimiento informado de los participantes y se respetó el resguardo a la confidencialidad

\section{Diseño}

Se utilizaron como técnicas de control de variables extrañas el bloqueo y la aleatorización. El procedimiento experimental consistió en formar grupos de sujetos homogéneos en la variable de bloqueo (categoría profesional: juveniles y aficionados) y dentro de cada bloque se asignó aleatoriamente la mitad de los participantes a cada condición de tratamiento (hipnosis y grupo control). De todas las personas que formaron parte del experimento se tomó una medida pretest de las variables dependientes antes de empezar a administrar los tratamientos y cuatro medidas postest.

\section{Variables}

La variable independiente objeto de estudio fue el procedimiento de la hipnosis. Las variables dependientes fueron las siguientes:

- Variables de naturaleza psicológica: (a) muy relajado-muy nervioso, (b) controlado-sin control, (c) muy enérgico-muy fatigado, (d) concentradodescentrado, (e) sin esforzarse-con gran esfuerzo. - Variables de naturaleza fisiológica: 1) Fatiga percibida, medida con la Escala de Borg (Borg, 1982). Se trata de una tabla con números entre 20 y 6 , colocados verticalmente y acompañados de valoraciones cualitativas entre fuerte, muy fuerte y muy, muy ligero. 2) Frecuencia cardiaca hasta no cumplir el criterio (mantenimiento de 70-80 pedaladas por minuto). 3) Covariables: Grado de sugestionabilidad.

\section{Instrumentos}

Los instrumentos utilizados fueron los siguientes:

1. Cuestionario de Identificación de Estados Psicológicos de Actuación (González, 1996), en el cual quedaban registrados los distintos estados psicológicos que los participantes experimentaron ese día durante la prueba de esfuerzo.

2. Escala de Sugestionabilidad de García-Cueto y Gordón (1998).

La escala utilizada en este estudio constaba de 10 ítems. A cada ítem bien realizado se le asignó el valor de 1 , siendo la mínima puntuación 0 y la máxima 10 puntos. Se ordenó a cada participante en función del grado de sugestionabilidad, así: alta (puntuación de 10 a 8), media (7 a 6), baja (0 a 5). Una sugestionabilidad alta e incluso media indicaría una buena capacidad de los participantes para cumplir el procedimiento.

\section{Escala de Fatiga Percibida de Borg (1982).}

Es una escala que relaciona la sensación del esfuerzo que percibe el deportista con un valor numérico. Se busca controlar el nivel de exigencia de la carga de entrenamiento y el grado de fatiga experimentado por el deportista durante la realización de la actividad deportiva.

4. Ordenador HP 1740.

5. Programa de ordenador Cardgirus Medical. En particular se utilizó el denominado Entrenamiento por Vatios, el cual aparece detalladamente descrito en el $o$.

6. Bicicleta de esfuerzo modelo Cardgirus.

\section{Procedimiento}

La secuencia de actuación llevada a cabo con los grupos experimentales fue la misma en ambos grupos, con la excepción de que el grupo control (GC) no recibió intervención con hipnosis. 
En primer lugar, se evaluó el grado de sugestionabilidad y luego se aplicó la prueba de esfuerzo con incrementos progresivos de la carga (Wasserman, 1988). Se realizaron cinco pruebas de esfuerzo con cada deportista a lo largo de cinco semanas. Esta prueba se aplicó en el pretest en ambos grupos y, en el caso del grupo experimental (GE), la realización de la misma tuvo lugar justo después de cada sesión de intervención con hipnosis.

Respecto al Test de Entrenamiento por Vatios, este comenzaba con 5 minutos de calentamiento sin carga a una cadencia de pedalada de 70-80 pedaladas minuto. Una vez pasados esos 5 minutos, se daban 2 minutos de reposo en los que el deportista se mantenía encima de la bicicleta sin pedalear. Transcurrido el tiempo de reposo, el deportista comenzaba a pedalear durante un minuto a 25 vatios entre 70-80 pedaladas. Cada minuto transcurrido se iba aumentando en 25 vatios, manteniéndose siempre la cadencia entre $70-80$ pedaladas. Pasado un cierto tiempo, y en función de las características de cada deportista, a consecuencia de la fatiga, no podía mantener la cadencia criterio. En ese momento el investigador contaba en voz alta de $10 \mathrm{a}$ $1 \mathrm{y}$, si durante esos 10 segundos el deportista no era capaz de volver a mantener la cadencia criterio de 70-80 pedaladas por minuto, la prueba de esfuerzo se daba por finalizada.

Este esquema de actuación fue el que se utilizó en todas las pruebas de esfuerzo que realizó el GC y en el pretest, el grupo experimental. En el GE, durante las sucesivas pruebas de esfuerzo (postest), cuando los deportistas no eran capaces de mantener la cadencia, se realizaba una cuenta atrás de cinco segundos y si en ese tiempo no volvían a la cadencia criterio, se introducía la sugestión poshipnótica, que se detalla adelante.

El procedimiento de hipnosis se realizaba inmediatamente antes de cada prueba de esfuerzo. La intervención, que estaba compuesta de tres partes, comenzaba con una técnica de relajación. Después venía una segunda parte denominada "profundización en hipnosis", de 20 minutos de duración, donde se introducían sugestiones de mayor relajación para que el deportista entrara en un estado de profunda hipnosis.
En el estado de hipnosis profunda se introdujo la sugestión poshipnótica. Se comenzó indicando exactamente el lugar donde dicha sugestión debía cumplirse, en este caso: "en la siguiente prueba de esfuerzo". También se aclaró el momento: "cuando tu mente consciente ya no pueda más y no consiga mantener la cadencia", quién iba a verbalizar la sugestión: "mi voz diciendo 1, 2, 3", lo que iba a ocurrir: "entonces entrarás de nuevo en un estado especial de profunda hipnosis, donde lo difícil se transforma en fácil" y qué se iba a activar "un estado donde tu inconsciente nuevamente se activará".

La sugestión poshipnótica se formuló con ciertas características especiales, buscando que los deportistas se dejaran llevar por el significado literal de los números 1, 2, 3: "mi voz diciendo 1, 2, 3 y muy probablemente un inconsciente transformando sensaciones desfavorables en sensaciones a favor. Pero también muy probablemente mi voz diciendo $1,2,3$ y un inconsciente asociando sensaciones desagradables con seguir manteniendo la cadencia de pedaleo y, a pesar de ello, ser capaz de continuar haciéndolo".

Las instrucciones del hipnotizador en la tercera parte de la intervención (fase de salida) mostraban a los participantes que podían salir sin ninguna dificultad del estado de hipnosis en el que se encontraban.

Al finalizar cada una de las pruebas de esfuerzo se les pasaba a todos los deportistas (GC y GE) la escala de Borg (Borg, 1982), anotándose la numeración elegida como percepción del esfuerzo. Justo después, estos complementaban el Cuestionario de Identificación de Estados Psicológicos de Actuación (González, 1996).

\section{Resultados}

\section{Análisis de los resultados}

Se realizaron análisis descriptivos de las variables de interés, observándose una mayor tendencia en el grupo experimental a puntuar positivamente en las variables de tipo psicológico (Tabla 1).

A pesar del número de participantes, se utilizaron pruebas paramétricas, debido a que todos los 
datos utilizados en el presente estudio cumplían las pruebas de normalidad $(p>0.05)$. Atendiendo a este criterio, se analizaron los datos mediante un análisis de varianza (ANOVA) 2 X 2, con 2 medidas (pretest y postest) como factor intrasujeto, $\mathrm{y}$ grupo de tratamiento (hipnosis y control) como factor entre-sujetos, cumpliéndose los supuestos de normalidad y homocedasticidad.

En las variables fatiga percibida y frecuencia cardiaca hasta no cumplir el criterio, se encontraron diferencias estadísticamente significativas en los efectos pretest-postest con un porcentaje de varianza explicada del $47 \%$, $28 \%$ y $30 \%$, respectivamente. La variable fatiga percibida presentaba un tamaño del efecto $\left(\eta^{2}\right)$ de 0.47 , es decir, un $47 \%$ de la variabilidad total de la VD se explicaba por los cambios producidos entre la medida pretest y postest. En las variables de tipo psicológico analizadas no se obtuvieron diferencias estadísticamente significativas en el análisis intrasujeto, entre-sujetos ni en la interacción entre ambas (Tabla 2).

La media global en fatiga percibida en las medidas pretest y postest fue respectivamente $16.45 \mathrm{y}$ $17.6(p=0.97)$ (Figura 1).

La frecuencia cardiaca hasta no cumplir el criterio entre el pretest y el postest fue 194.1 y 190.4 $(p=0.64)$ respectivamente, presentando un tamaño del efecto de 0.28 (Figura 2).

\section{Discusión}

En este estudio se evaluó la eficacia de la hipnosis en la modificación de una serie de variables psicológicas y fisiológicas durante la realización de pruebas de esfuerzo en ciclistas juveniles y aficionados. En este sentido, algunas investigaciones han comprobado la posibilidad de modificar ciertas variables

\section{TABLA 1}

Estadísticos descriptivos

\begin{tabular}{|c|c|c|c|c|}
\hline \multirow{2}{*}{ Variable Dependiente } & \multicolumn{2}{|c|}{ Grupo experimental } & \multicolumn{2}{|c|}{ Grupo control } \\
\hline & $M$ & $\mathrm{DE}$ & $M$ & $D E$ \\
\hline \multicolumn{5}{|l|}{ Fatiga percibida (Borg) } \\
\hline PRETEST & 16.7 & 1.41 & 16.2 & 1.6 \\
\hline POSTEST & 17.8 & 1.31 & 17.4 & 0.91 \\
\hline \multicolumn{5}{|l|}{ Vatios hasta no cumplir el criterio } \\
\hline PRETEST & 372.5 & 39.9 & 375 & 54.1 \\
\hline POSTEST & 382.5 & 75.6 & 378 & 91.1 \\
\hline \multicolumn{5}{|l|}{ Muy relajado-Muy nervioso } \\
\hline PRETEST & 3.7 & 1.34 & 3.9 & 1.12 \\
\hline POSTEST & 2.8 & 0.63 & 3.8 & 1.4 \\
\hline \multicolumn{5}{|l|}{ Controlado-Sin control } \\
\hline PRETEST & 3.6 & 1.1 & 3 & 1.2 \\
\hline POSTEST & 2.7 & 1.05 & 2.9 & 1.12 \\
\hline \multicolumn{5}{|l|}{ Muy enérgico-Muy fatigado } \\
\hline PRETEST & 4.2 & 1.1 & 4.1 & 1.2 \\
\hline POSTEST & 3.2 & 1.6 & 4 & 1.3 \\
\hline \multicolumn{5}{|l|}{ Concentrado-Descentrado } \\
\hline PRETEST & 4.3 & 1.05 & 3 & 1.7 \\
\hline POSTEST & 3.4 & 0.81 & 2.1 & 0.51 \\
\hline \multicolumn{5}{|l|}{ Sin esforzarse-Con gran esfuerzo } \\
\hline PRETEST & 4.7 & 0.95 & 4.6 & 1.4 \\
\hline POSTEST & 3.7 & 1.5 & 5 & 1.19 \\
\hline
\end{tabular}

Fuente: elaboración propia. 
TABLA 2

Análisis de la varianza

\begin{tabular}{llcccc}
\hline \multicolumn{1}{c}{ Variable Dependiente } & \multicolumn{1}{c}{ Efectos } & $F$ & $g l$ & $p$ & h2 \\
\hline Fatiga percibida (Borg) & Efectos Pretest-Postest & 13.66 & 1.16 & $0.002 *$ & 0.47 \\
& Efectos Tratamientos & 0.002 & 1.16 & 0.97 & 0.00 \\
& Efectos Interacción & 0.61 & 1.16 & 0.44 & 0.38 \\
\hline Frecuencia cardiaca & Efectos Pretest-Postest & 6.18 & 1.16 & $0.024 *$ & 0.28 \\
no cumplir criterio & Efectos Tratamientos & 0.21 & 1.16 & 0.64 & 0.013 \\
& Efecto Interacción & 0.52 & 1.16 & 0.48 & 0.032 \\
\hline Muy relajado-Muy nervioso & Efectos Pretest-Postest & 1.9 & 1.16 & 0.19 & 0.105 \\
& Efectos Tratamientos & 0.99 & 1.16 & 0.33 & 0.059 \\
& Efecto Interacción & 0.99 & 1.16 & 0.33 & 0.059 \\
\hline Controlado-Sin control & Efectos Pretest-Postest & 1.34 & 1.16 & 0.26 & 0.077 \\
& Efectos Tratamientos & 2.35 & 1.16 & 0.14 & 0.13 \\
& Efecto Interacción & 0.04 & 1.16 & 0.83 & 0.003 \\
\hline Muy enérgico-Muy fatigado & Efectos Pretest-Postest & 2.44 & 1.15 & 0.14 & 0.14 \\
& Efectos Tratamientos & 1.48 & 1.15 & 0.24 & 0.09 \\
& Efecto Interacción & 0.41 & 1.15 & 0.53 & 0.027 \\
\hline Concentrado-Descentrado & Efectos Pretest-Postest & 1.56 & 1.16 & 0.22 & 0.089 \\
& Efectos Tratamientos & 1.56 & 1.16 & 0.22 & 0.089 \\
& Efecto Interacción & 4.28 & 1.16 & 0.22 & 0.089 \\
\hline Sin esforzarse-Con gran esfuerzo & Efectos Pretest-Postest & 0.55 & 1.16 & 0.46 & 0.034 \\
& Efectos Tratamientos & 2.69 & 1.16 & 0.12 & 0.14 \\
& Efecto Interacción & 1.96 & 1.16 & 0.18 & 0.10 \\
\hline
\end{tabular}

Nota. El asterisco señala que el efecto ha sido estadísticamente significativo al nivel de confianza del $95 \%$.

Fuente: elaboración propia.

psicológicas relacionadas con el deporte, mediante el uso de la hipnosis (Barker et al., 2010; Fernández, Fidalgo, Zurita, García \& Sánchez, 2009; Grindstaff \& Fisher, 2006).

No existieron diferencias estadísticamente significativas entre los grupos, aunque sí en los efectos pretest-postest $(p=0.002$ en fatiga percibida y 0.024 en frecuencia cardiaca hasta no cumplir el criterio), en ninguna de las variables de tipo fisiológico registradas a lo largo de las sesiones en relación a las medidas pretest $(p=0.97$ en fatiga percibida y 0.64 en frecuencia cardiaca hasta no cumplir el criterio). Dicho de otra forma, no hubo ninguna variable fisiológica que indicara que uno de los grupos se encontraba en mejores condiciones físicas para afrontar las pruebas de esfuerzo. En este sentido, se puede entender que los resultados obtenidos en las variables de tipo psicológico, donde tampoco se obtuvieron diferencias estadísticamente significativas, no se vieron afectadas por el posible estado de forma deficitario de un grupo respecto al otro. En la misma línea, son numerosos los estudios que han investigado la relación entre el estado de forma del deportista a nivel físico y fisiológico y los factores psicológicos (Johnson \& Ivarsson, 2010; Ortín, Garcés de los Fayos \& Olmedilla, 2010).

Teniendo en cuenta la influencia de las variables psicológicas en el contexto deportivo (Eraña, 2004; Olmedilla, Ortega, Prieto \& Blas, 2009; Olmedilla, Prieto \& Blas, 2010, 2011; Vallejo, 2004), se evaluó el posible efecto de la hipnosis en la modificación de todas aquellas variables psicológicas que aparecen registradas en el Cuestionario de Lorenzo. Los resultados indicaron que la hipnosis no era una técnica que influía en la modificación de estos estados psicológicos durante la realización de pruebas de esfuerzo. Pero sí es cierto que la hipnosis utilizada en el grupo experimental favoreció la obtención de puntuaciones más positivas en estas variables. Siguiendo con lo expuesto, cabría recordar que la 


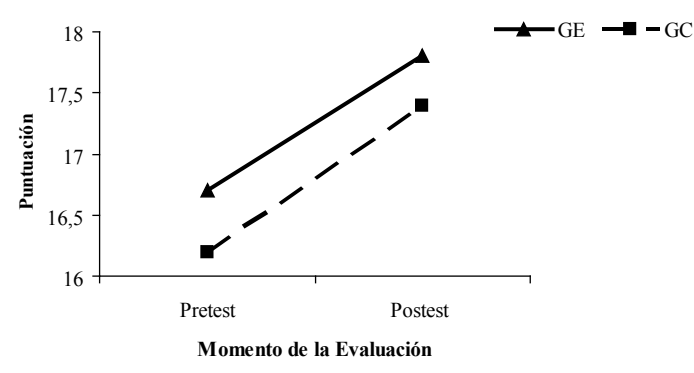

Figura 1. Fatiga percibida (Borg).

Fuente: elaboración propia.

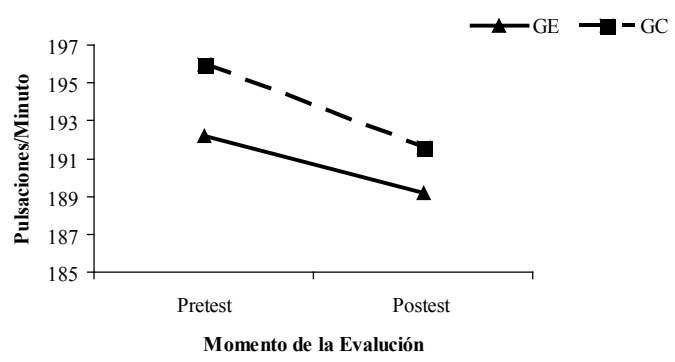

Figura 2. Frecuencia cardiaca hasta no cumplir el criterio (70 pedaladas por minuto).

Fuente: elaboración propia.

intervención contaba inicialmente con una técnica de relajación, la cual fue aplicada sucesivamente en un total de cuatro sesiones. En este sentido, pudo darse una generalización de este aprendizaje, es decir, el deportista pudo saber cómo relajarse o estar menos fatigado durante las pruebas de esfuerzo, gracias a un conocimiento previo. A este respecto, no se debe olvidar el efecto que tienen las técnicas de relajación en la modificación de variables como la ansiedad o la fatiga (Acosta, 2007).

Atendiendo a lo expuesto, se debe dar cuenta de que la intervención contenía un gran número de elementos favorecedores de la capacidad de concentración y focalización de la atención. En este sentido, se entiende que la intervención pudo propiciar que los participantes a la largo de cuatro sesiones, centraran cada vez más la atención en todo un conjunto de sensaciones internas, inten- sificando de esta forma la atención hacia ciertos estímulos propioceptivos y favoreciendo de esta forma cambios en las variables psicológicas analizadas. Respecto a lo comentado, son numerosos los estudios que ofrecen pruebas claras de la relación existente entre prestar atención a las sensaciones internas y capacidad de control y concentración personal (Goldstein \& Korfield, 2007).

Por otro lado, no se debe descartar la posible interrelación e influencia entre las distintas variables de tipo psicológico, es decir, es probable que si el deportista sentía que en su ejecución estaba concentrado, controlado y sin tensión, esta situación pudiera afectar también y producir cambios en otros estados relacionados, por ejemplo confianza, falta de esfuerzo mental. La literatura ofrece pruebas de la relación e interdependencia existente entre los factores psicológicos (Gould \& Weinberg, 2010; Martin, 2008).

Referente al grupo control, la mayoría de las variables psicológicas se mantuvieron estables e incluso en alguna de ellas las puntuaciones empeoraron. Se entiende que estos resultados pudieron deberse a que los deportistas de este grupo no se vieron favorecidos por la aplicación del procedimiento hipnótico.

Otro aspecto a reseñar en este estudio tiene que ver con ciertas limitaciones que se deben tener en cuenta en futuras investigaciones. Se deberían, en primer lugar, replicar estudios similares con mayor número de participantes, incluyendo a deportistas de diferentes modalidades.

Por último, sería de interés incrementar el número de sesiones de intervención, con el fin de saber si el procedimiento de hipnosis habría favorecido la obtención de diferencias estadísticamente significativas en relación con las variables de tipo psicológico registradas.

\section{Referencias}

Acosta, J. M. (2007). Gestión eficaz del tiempo y control del estrés. Madrid: Esic.

Barker, K. \& Jones, M. (2006). Using hypnosis, technique refinement and self-modeling to enhance 
self-efficacy: A case study in cricket. Sport Psychologist, 20(1), 94-110.

Barker, J., Jones, M. \& Greenlees, I. (2010). Assessing the immediate and maintained effects of hypnosis on self-efficacy and soccer wall-volley performance. Journal of Sport and Exercise Psychology, 32(2), 243-252.

Borg, G. (1982). Psychophysical bases of perceived exertion. Medicine and Science in Sports and Exercise, 14(5), 337-381.

Eraña, I. (2004). Entrenamiento psicológico con jóvenes tenistas. Revista de Psicología del Deporte, 13(2), 263-271.

Fernández, R., Secades, R., Terrados, N., García, E. \& García, J. M. (2004). Efecto de la hipnosis y la terapia de aceptación y compromiso en la mejora de la fuerza física en piragüistas de élite. Revista Internacional de Psicología Clínica y Salud, 4(3), 481- 493.

Fernández, R., Fidalgo, A. M., Zurita, F., García, J. M. \& Sánchez, L. (2009, 27 de febrero). Efectos de la hipnosis en la mejora de variables físicas y psicológicas dentro del contexto del deporte. Revista Electrónica de Portales Médicos.com, 4(4), 40. Disponible en http://www.portalesmedicos.com/publicaciones/ articles/1389/1/Efectos-de-la-hipnosis-en-la-mejora-de-variables-fisicas-y-psicologicas-dentro-delcontexto-del-deporte.html

García-Cueto, E. \& Gordón, O. (1998). Elaboración y análisis de una Escala de Sugestionabilidad. Psicología Conductual, 6(3), 519-531.

García-Naveira, A. \& Remor, E. (2011). Motivación de logro, indicadores de competitividad y rendimiento en un equipo de deportistas (fútbol) de competición varones entre 14 y 24 años. Universitas Psychologica, 10(2), 477-487.

Goldstein, J. \& Korfield, K. (2007). Vipassana. El camino para la meditación interior. Barcelona: Kairós.

Gould, G. \& Weinberg, R. (2010). Fundamento de psicología del deporte y del ejercicio físico. Madrid: Panamericana.

González, J. (1996). El entrenamiento psicológico en los deportes. Madrid: Biblioteca Nueva.

Grindstaff, J. S. \& Fisher, L. A (2006). Sport psychology consultants' experience of using hypnosis in their practice: An exploratory investigation. Sport Psychologist, 20(3), 368-386.
Johnson, U. \& Ivarsson, A. (2010). Psychological predictors of injuries among junior soccer players. Scandinavian Journal of Medicine $\mathcal{E}$ Science in Sport, 21(1), 129-136. doi: 10.1111/j.1600-838.2009.01057.x

Jara, P. \& Martínez, F. (1999). Hipnosis, hipnotizabilidad y expectativas de respuesta: una revisión crítica. Anales de Psicología, 15(1), 39-56.

Kirenskaya, A. V., Novotosky, V. \& Zvonikov, V. M (2011). Waking EEG spectral power and coherence differences between high and low hypnotizable. International Journal of Clinical and Experimental Hypnosis, 59(4), 441-453. doi: 10.1080/00207144.2011.594744

Landolt, A. \& Milling, S. (2011). The efficacy of hypnosis as an intervention for labor and delibery pain: A comprehensive methodological review. Clinical Psychology Review, 31(6), 1022-1031. doi: 10.1016/j. cpr.2011.06.002

Lindsay, P., Maynard, I. \& Thomas, O. (2005). Effects of hypnosis on flow states and cycling performance. Sport Psychologist, 19(2), 164-177.

Lorenzo, J., Pujals, C., Navarro, R. \& Lorenzo, A. (2012). Análisis de los efectos de un programa de intervención psicológica en jóvenes jugadores de baloncesto. Revista de Psicología del Deporte, 21(1), 43-8.

Martin, G. (2008). Psicología del deporte. Guía práctica del análisis conductual. Madrid: Prentice-Hall.

Moreno, J. A., Conte, L., Borges, F. \& González-Cutre, D. (2008). Necesidades psicológicas básicas, motivación intrínseca y propensión a la experiencia autotélica en el ejercicio físico. Revista Mexicana de Psicología, 25(2), 305-312.

Olmedilla, A., Ortega, E., Prieto, J. M. \& Blas, A. (2009). Percepción de los tenistas respecto a los factores que pueden provocar lesiones: diferencias entre federados y no federados. Cuadernos de Psicología del Deporte, 9(2), 7-18.

Olmedilla, A., Prieto, J. M. \& Blas, A. (2010). Lesiones en tenistas: percepción subjetiva sobre la importancia de los factores causales. Revista Internacional de Medicina de Ciencias de la Actividad Física y el Deporte, 10(38), 323-335.

Olmedilla, A., Prieto, J. M. \& Blas, A. (2011). Relaciones entre el estrés psicosocial y lesiones deportivas en tenistas. Universitas Psychologica, 10(3), 909-22. 
Ortín, F. J., Garcés de los Fayos, E. J. \& Olmedilla, A. (2010). Influencia de los factores psicológicos en las lesiones deportivas. Papeles del Psicólogo, 31(3), 143-154.

Pates, F., Maynard, I. \& Cummings, A. (2002). The effects of hypnosis on flow states and three-point shooting performance in basketball players. Sport Psychologist, 16(1), 34-47.

Pérez, M. (1999). Teoría dramatúrgica de la hipnosis. Anales de Psicología, 15(1), 27-38.
Raz, L., Uriel, H. \& Shany, E. (2011). Hypnotizability and sensorimotor gating: A dopalinergic mechanism of hypnosis. International Journal of Clinical and Experimental Hypnosis, 59(4), 399-405. doi: 10.1080/00207144.2011.594678

Vallejo, M. (2004). Intervención psicológica en saltos de trampolín. Revista de Psicología del Deporte, 13(1), 95-115.

Wasserman, K. (1988). Principles of exercise testing and interpretation (2a. ed.). Philadelphia: Lea \& Febiger. 
